\title{
Biomechanical comparison between the custom-made mandibular condyle prosthesis and total temporomandibular joint prosthesis in finite element analysis
}

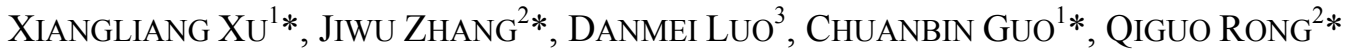 \\ ${ }^{1}$ National Engineering Laboratory for Digital and Material Technology of Stomatology, Department of Oral and Maxillofacial Surgery, \\ Peking University School and Hospital of Stomatology, Beijing, China. \\ ${ }^{2}$ Department of Mechanics and Engineering Science, College of Engineering, Peking University, Beijing, China. \\ ${ }^{3}$ Beijing Machine and Equipment Institute, Beijing, China.
}

\begin{abstract}
Purpose: The purpose of this study was to compare the biomechanical behavior of the custom-made mandibular condyle prosthesis and total TMJ prosthesis. Methods: Three models of one beagle dog, the condyle prosthesis (Model 1, replacing the right condyle only), the TMJ prosthesis (model 2, replacing the whole right TMJ) and the intact TMJ (model 3) were established, and the mechanical responses under muscle forces loading were analyzed using finite element method. Results: Models 1 and 3 had the similar stress distribution on the right disc, which suggested that the condyle prosthesis did not change the disc stress so much when the muscle forces were applied. The stress of the right TMJ prosthesis in Model 2 was larger than both Models 1 and 3, and the stress of the contralateral TMJ reduced by $12 \%$ in Model 2 . The anterior border of the condyle seemed to be a stress concentration region, not only for the intact condyle, but also for the condyle prosthesis and the total TMJ prosthesis. Conclusions: The total TMJ prosthesis changed the biomechanical balance of the bilateral TMJ. When the condyle prosthesis iss applied, the custom-made profile is recommended.
\end{abstract}

Key words: temporomandibular joint, condyle prosthesis, total temporomandibular joint prosthesis, finite element analysis

\section{Introduction}

The temporomandibular joint (TMJ) is a very complex joint with variable functions, which plays a crucial role in our daily speech, expression and chewing. Trauma, infection, ablation of tumor, ankylosis and idiopathic resorption could cause TMJ defects, which should be reconstructed properly. The reconstruction of acquired TMJ defects is an obvious challenge for oral and maxillofacial surgeons [26]. TMJ discs between the condyle and the fossa-eminence play very important roles, such as distributing joint forces and stabilizing the mandible [5]. During functions, the discs show a viscoelastic response to static and dynamic loading and absorbs stress [1], [22]. For the condyle prosthesis, the disc functions as a cushion. If the disc was removed during surgery, the resorption of the bone could occur under the stress [25]. Besides, changes of loading style after the reconstruction surgery cause the stress changes for bilateral TMJ discs and might cause calcifications [10]. Therefore, contralateral TMJ stress should be considered carefully when the prostheses were applied in unilateral TMJ.

Compared to autogenous reconstruction developed over the decades, alloplastic TMJ prostheses can reduce donor site morbidity, as well as provide immediate function and be customizable [6], [16], [19], [27].

* Corresponding authors: Chuanbin Guo, National Engineering Laboratory for Digital and Material Technology of Stomatology, Department of Oral and Maxillofacial Surgery, Peking University School and Hospital of Stomatology, Beijing, China, e-mail: guodazuo@vip.sina.com; Qiguo Rong, Department of Mechanics and Engineering Science, College of Engineering, Peking University, Beijing, China. E-mail: qrong@pku.edu.cn; Xiangliang Xu, National Engineering Laboratory for Digital and Material, Technology of Stomatology, Department of Oral and, Maxillofacial Surgery, Peking University School and Hospital of Stomatology, Beijing, China; Jiwu Zhang, Department of Mechanics and Engineering Science, College of Engineering, Peking University, Beijing, China.

Received: September 1st, 2020

Accepted for publication: October 21st, 2020 
Based on the location of TMJ defect, alloplastic TMJ reconstruction could be divided into 3 models: prostheses for the singular replacement of the fossa, prostheses and reconstruction plates for singular replacement of the condyle and prostheses for combined fossa-condyle replacement [4]. Each kind of prostheses should be applied according to different indications. When total bony ankylosis of TMJ is the case, the third mode of prostheses is more appropriate. When the disc is intact, prostheses for singular replacement of the condyle are more suitable than other prostheses.

The mandibular body connects the left and right joints. Disability or limitation of functional movements in one side definitely affects the contralateral TMJ. When one kind of prostheses is applied unilaterally, the actions of contralateral TMJ should receive more attention. Our previous study proposed a custom-made TMJ prosthesis fabricated by selective laser melting [28]. We found that the magnitude of the compressive strain on the condyle neck of the mandible with the prosthesis was lower than that on the condyle neck of the intact mandible with the exception of the area around the screws, where muscle forces were applied. Besides our research, some studies had evaluated the TMJ prosthesis including standard and custom-made design with finite element analysis (FEA) [17], [20]. No study comparing the biomechanical behaviors of the condyle prosthesis, the total TMJ prosthesis and the intact TMJ has been found till now. In the present study, we compared the biomechanical responses of three models with FEA, including the contralateral TMJ stress when the muscle forces were applied, since FEA has been used to research biomechanical behavior of the TMJ for a long time [3], [12], [20], [21]. In the next stage of research, the customized condyle prosthesis and the total TMJ prosthesis for different dogs are planned to be manufactured as reported in this study, and in vivo experiments are to be performed to check their effectiveness and compare contralateral TMJ changes.

\section{Materials and methods}

All experiments were authorized by Peking University Institutional Review Board (LA2014244). The bone structure morphology of the TMJ was obtained from an adult beagle dog. The dog was obtained from Department of Laboratory Animal Science, Peking University Health Science Center. The dog was individually housed in a stainless-steel cage, maintained on alternating 12-h cycles of light and dark and pro- vided with normal canine diet. A veterinarian performed the examination and confirmed it was in good health before computed tomography (CT) scans. CT scans were performed under general anesthesia using an 8-slice scanner (BrightSpeed, GE Medical Systems, USA) with a rotation time of $1 \mathrm{sec}$, a slice thickness of $1.25 \mathrm{~mm}$, voltage of $120-140 \mathrm{kV}$ and automatic exposure control. After CT scans, the dog recovered from general anesthesia and continued living as before.

CT images were taken for four positions of the mandible: the closed, 1/3 open, $2 / 3$ open, and widest open positions. As shown in Fig. 1, three geometric models of the mandible and TMJ complex in both sides were established based on these CT images: the condyle prosthesis replacing the right TMJ (Model 1), the total TMJ prosthesis replacing the whole right TMJ (Model 2) and the intact TMJ (Model 3). All three models were imported into the finite element software ANSYS for stress analysis. The TMJ prostheses can then be manufactured by Mlab Cusing 3D Metal Printing Machine (Concept Laser, Lichtenfels, Germany) and applied in future animal experiments.
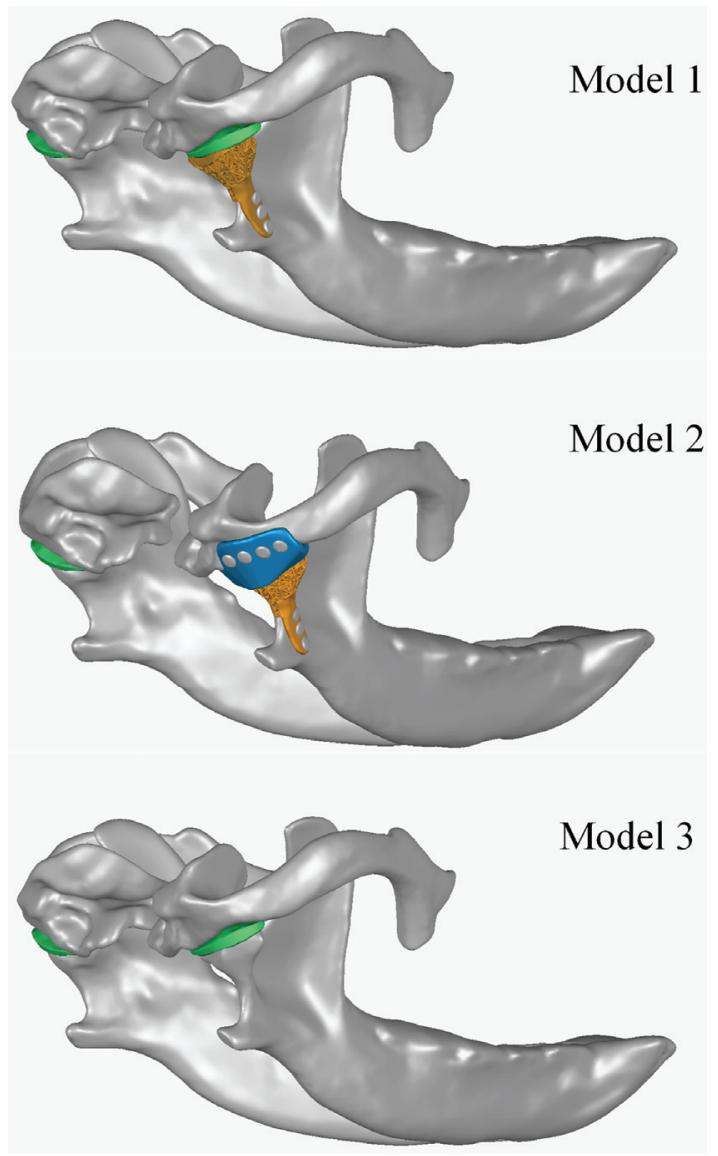

Fig. 1. The condyle prosthesis replacing the right condyle (Model 1), the total TMJ prosthesis replacing the whole right TMJ (Model 2), the intact TMJ (Model 3) 


\subsection{The model with the condyle prosthesis replacing the right TMJ only}

In this model, the condyle prosthesis was designed accordingly to the method developed previously [28]. To put it brief, the condyle prosthesis was fixed to the mandible with 4 screws. The screws and the condyle component were made of titanium- 6 aluminum- 4 vanadium alloy (Ti-6Al-4V). The profile of the prosthesis was based on the anatomical shape of the beagle's right condyle. On the top of the condyle prosthesis, a 1-mm layer of $1 \mathrm{~mm}$ with a polished outer surface was kept as the articular surface of the condyle. The combined fixation structures for the condyle prosthesis contained an inlay rod and an onlay plate to strengthen the connection between the prosthesis and the remaining mandible. The tetrahedron structural design with the mean cell size of $2500 \mu \mathrm{m}$ and strut diameter of $570 \mu \mathrm{m}$ of open-porous titanium scaffold was applied to fabricate the middle part of the condyle prosthesis. The disc and the cranial fossa were reconstructed according to the CT images.

\subsection{The model with the total TMJ prosthesis replacing the whole right TMJ}

The total TMJ prosthesis was constructed according to our previous study [28]. In short, it was composed of condyle and fossa components, which were fixed to the mandible and zygoma with screws separately. The condyle component was constructed as the condyle prosthesis above. The fossa component was made of ultrahigh-molecular-weight polyethylene (UHMWPE). The lower surface of the fossa component was obtained by kinematic analysis of condyle movement, which was considered as the envelope surface. The upper surface of the fossa component, which was also considered as contact interface, was based on skull geometry with a best-fit position in the bone. The space between the envelope surface and the contact interface was where the fossa component occupied.

\subsection{The intact $\mathrm{TMJ}$ model}

The right TMJ in the intact TMJ model and the contralateral TMJs of all three models were constructed according to the CT images. The CT data were imported into MIMICS (Version 10.01, Materialise, Inc., Leuven, Belgium) to reconstruct its geometry.

\subsection{Finite element models}

All three models were analyzed with the use of FEA software ANSYS. The teeth were removed in these models because they were considered to have marginal influence on the biomechanics of the mandible, especially on the behavior of the condyles, as shown in previous studies [2], [13], [16]. Only the part of the skull connecting the fossa component and the bilateral zygomatic arches were modeled for simplicity.

All materials in this study were considered isotropic and homogeneous. The material properties of the models were assigned on the basis of previous studies (Table 1) [11], [16], [17]. The Young's modulus was $12.8 \mathrm{GPa}$ for the mandible cortical bone and $114 \mathrm{GPa}$ for the condyle prosthesis and screws, respectively [11]. Poisson ratios for all hard tissues were assumed to be 0.3 .

Table 1. Material properties in the FEA models

\begin{tabular}{|l|l|c|c|}
\hline \multicolumn{1}{|c|}{ Model } & \multicolumn{1}{|c|}{ Material } & $\begin{array}{c}\text { Young's } \\
\text { modulus } \\
{[\mathrm{MPa}]}\end{array}$ & $\begin{array}{c}\text { Poisson's } \\
\text { ratio }\end{array}$ \\
\hline Skull & Cortical bone & 12.800 & 0.3 \\
\hline Mandible & Cortical bone & 12.800 & 0.3 \\
\hline Condyle component & Titanium alloy & 114.000 & 0.3 \\
\hline Screws & Titanium alloy & 114.000 & 0.3 \\
\hline Fossa component & UHMWPE & 500 & 0.3 \\
\hline Articular disc & Fibrocartilage & 47.1 & 0.4 \\
\hline
\end{tabular}

The top surfaces of the skull and the ends of the zygomatic arch were fully constrained from movement in all directions. The loads were applied on both sides of the mandible via corresponding muscle forces. All data relevant to muscle forces were obtained from the study by $\mathrm{Hu}$ et al. [8]. The location of the muscular attachments and the direction of muscle forces were defined from anatomical data.

The articular disc with approximately cylindrical shape was manually created based on the radiology images of dog's skull and the previous study [14]. The upper and lower surfaces of the articular disc contacted with the skull and mandible. The interface between the bone and the articular disc was modeled as surface-to-surface contact with a friction coefficient of 0.001 [21].

For the prostheses in Models 1 and 2, the contact interfaces of the screws-prosthesis and screws-bone 
were considered bonded. The interfaces between the prosthesis surface and the bone surface were modeled as surface-to-surface contact with a friction coefficient of 0.3 , based on published studies [16], [18]. The friction ratio of the interfaces between the fossa component and the condyle component, the disc and the condyle prosthesis, was considered to be 0.01 [28].
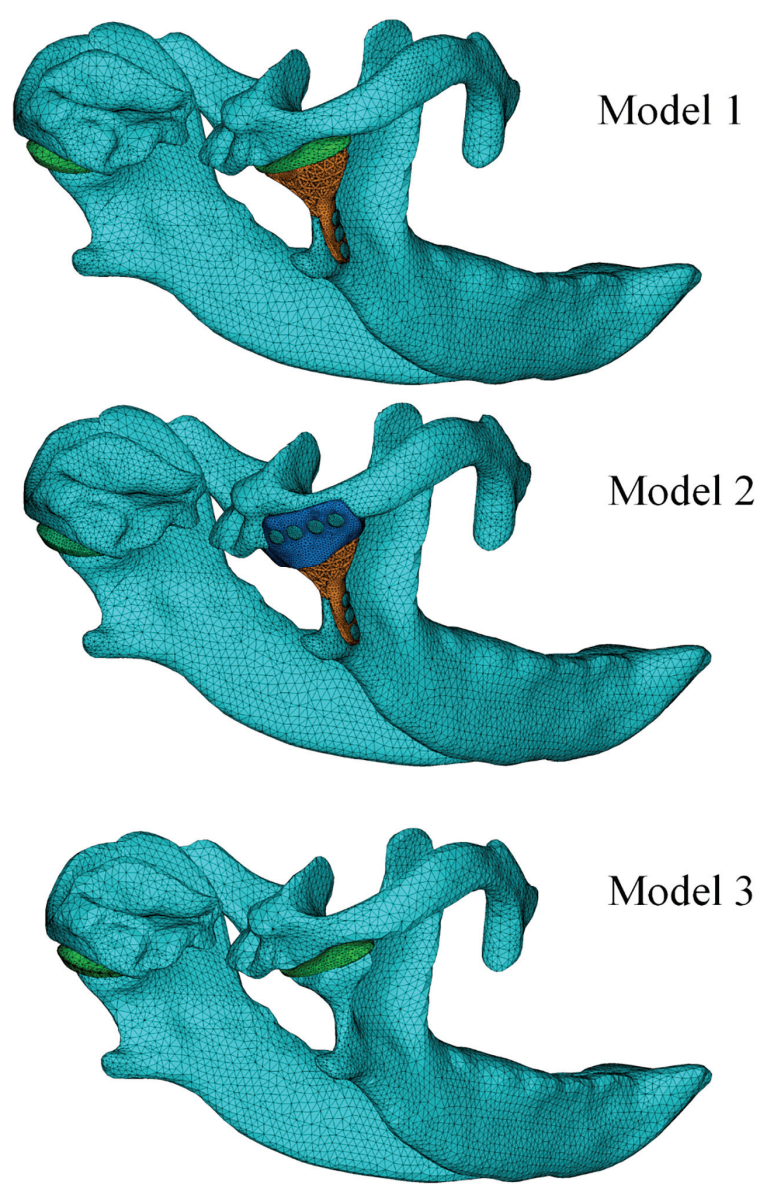

Fig. 2. Finite element models for the condyle prosthesis (Model 1), the total TMJ prosthesis (Model 2) and the intact TMJ (Model 3)

The bone, articular disc, implant and screws were meshed with tetrahedral elements, as these elements were well suited to the mesh irregular and complex geometries. The scaffolds were meshed with beam elements and each strut was a single unit. Aimed to the satisfied convergence of the numerical results, the meshes of the models were dense enough shown in Fig. 2. The number of cells and nodes was shown in Table 2.

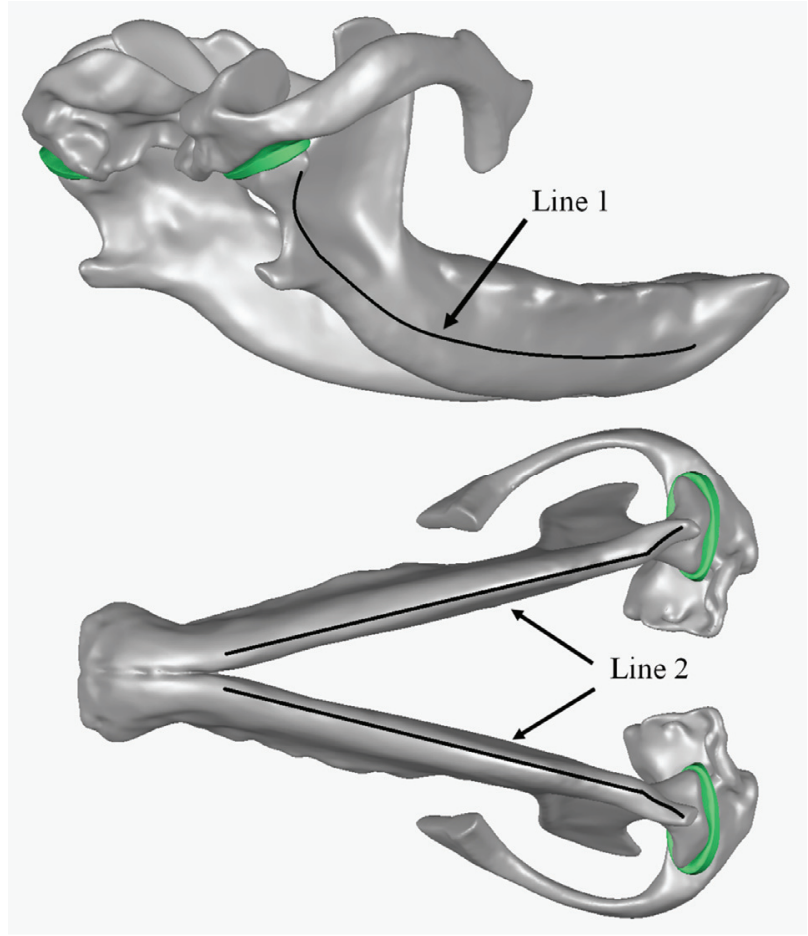

Fig. 3. The location of two control lines

After the muscle forces were applied on the FEA models, the biomechanical properties of 3 models were investigated, and the behavior of the discs, the prostheses and the mandibles were compared. To analyze the strain on the external surface of the mandible, two different lines were chosen (Fig. 3). Line 1 traversed characteristic structures presented on the external surface of the mandible. Line 2 extends from the right condyle to the left condyle along the lower border of the mandible.

Table 2. Number of elements and nodes in the FEA models

\begin{tabular}{|l|c|c|c|c|c|c|}
\hline & \multicolumn{3}{|c|}{ Element } & \multicolumn{3}{c|}{ Nodes } \\
\hline & Model 1 & Model 2 & Model 3 & Model 1 & Model 2 & Model 3 \\
\hline Bone & 123715 & 312637 & 224817 & 65828 & 83154 & 24552 \\
\hline Prostheses & 15552 & 55981 & & 4129 & 13304 & \\
\hline Screws & 6988 & 35753 & & 1702 & 8019 & \\
\hline Right articular discs & 1607 & & 1607 & 3211 & & 3211 \\
\hline Left articular discs & 1613 & 1613 & 1613 & 3220 & 3220 & 3220 \\
\hline Scaffolds & 1992 & 1992 & & 1467 & 1467 & \\
\hline
\end{tabular}




\section{Results}

\subsection{Behavior of 5 dises and 1 fossa component}

In the three models, a total of 5 TMJ discs were reconstructed and the stresses on them were calculated after the muscle forces were applied on the models. The fossa component in Model 2 was also compressed as other 5 TMJ discs when the mandible functioned. Therefore, the stress of all 6 structures including 5 TMJ discs and 1 fossa component was compared in our study (Figs. 4, 5; Table 3). The results showed that the difference of the stress on the disc in Models 1 and 3 was around 5\%. The maximum von Mises
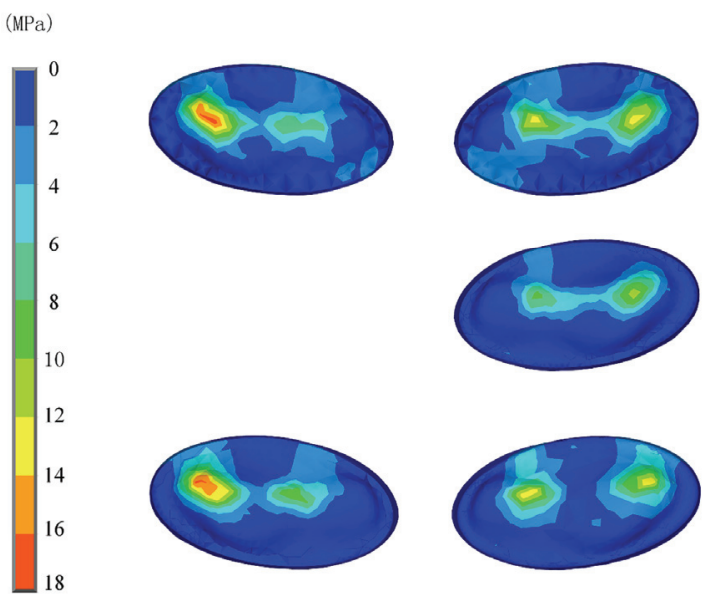

Model 1
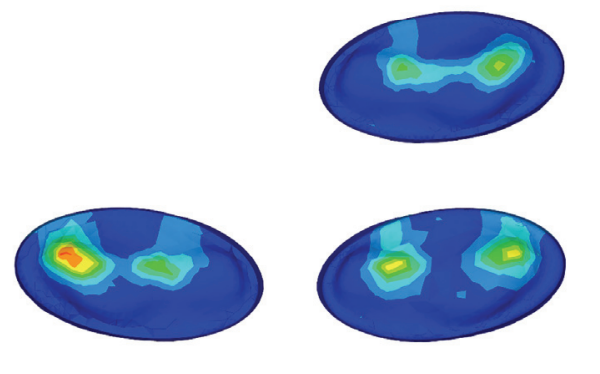

Model 2 Right Disc

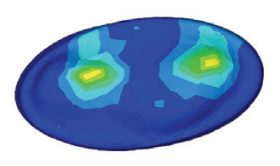

Model 3

Fig. 4. Von Mises stress distributions of five discs
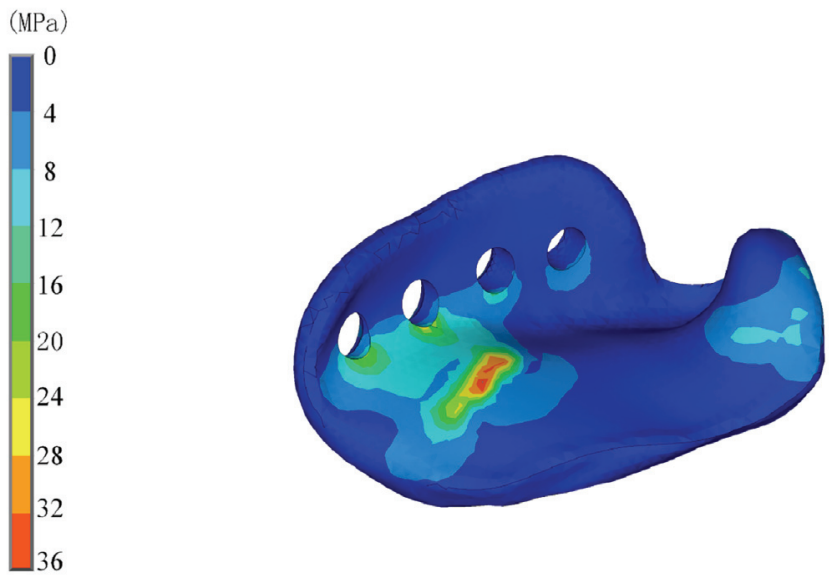

Fig. 5. Von Mises stress distribution of the fossa component of the TMJ prosthesis replacing the right TMJ in Model 2 stress in the right disc of Model 1 was by 5.7\% larger than that in Model 3, while the stress in the left disc of Model 1 was by $3.1 \%$ lesser than that in Model 3. As shown in Fig. 5, the maximum von Mises stress in the fossa component of Model 2 was $36.9 \mathrm{MPa}$, by $113.8 \%$ larger than that in model 1 and by $126.1 \%$ larger than that on Model 3. The stress in the left disc of Model 2 was $11.91 \mathrm{MPa}$, by $12.0 \%$ lesser than that in Model 1 and by $14.7 \%$ lesser than that in Model 3 (Table 3). The von Mises stress of the fossa component was much larger than that of 5 discs, and the stress distribution of all 6 structures were similar (Figs. 4, 5; Table 3).

Table 3. Maximum von Mises stress of discs and the fossa component of the total TMJ prosthesis [MPa]

\begin{tabular}{|l|c|c|c|}
\hline & Model 1 & Model 2 & Model 3 \\
\hline $\begin{array}{l}\text { Right joint discs/ } \\
\text { fossa component }\end{array}$ & 17.26 & 36.9 & 16.32 \\
\hline Left joint discs & 13.54 & 11.91 & 13.97 \\
\hline
\end{tabular}

\subsection{Behavior of condyle components}

The stress distributions of the condyle prosthesis in Model 1 and the condyle component in Model 2 were similar as shown in Fig. 6. The stress concentration region of both models laid in the lateral part of the prostheses. However, the maximum von Mises stress in Model 2 was larger than that in Model 1. They were 156.2 MPa in Model 2 and 134.2 MPa in Model 1, respectively.
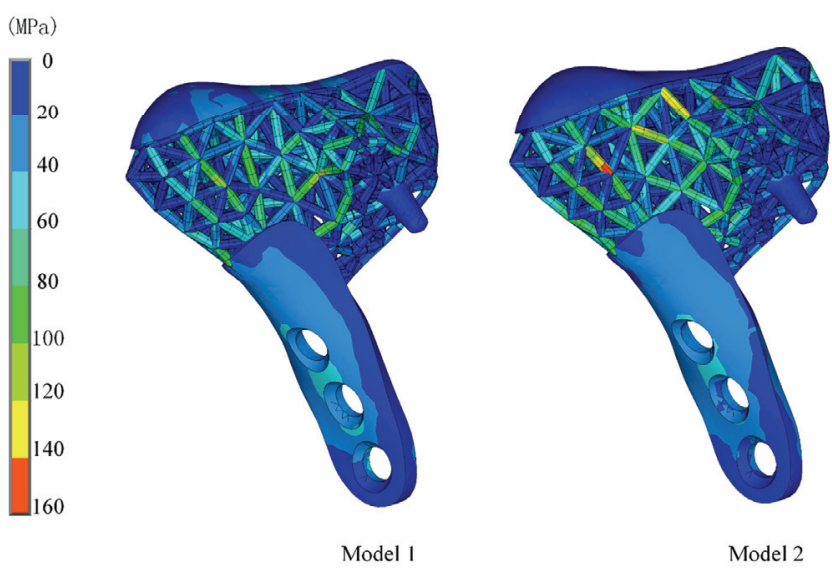

Fig. 6. Von Mises stress distributions of the condyle prosthesis in Model 1 and the condyle component of the TMJ prosthesis in Model 2 


\subsection{Behavior of mandibles}

In Figures 7 and 8, the distribution of the maximum and minimum principal strains was shown. The maximum and minimum principal strains along two lines on the mandible were shown in Figs. 9-12. In Model 3, the maximum and minimum principal strains in the intact mandible presented a symmetrical distribution in the regions of the bilateral condyles. For line 1 , the maximum principal strains in the right mandible in Model 1 and Model 2 were larger than that in the left mandible. The condyle prosthesis and the condyle component of the total TMJ prosthesis changed the strain of the right mandible and presented larger strain compared to the left. The minimum principal strain distribution of the right condylar region in Model 2 was significantly different from other models since the total TMJ prosthesis replaced the right TMJ. For line 2, the strain difference between the right condyle and the left condyle among the three models was not so obvious.

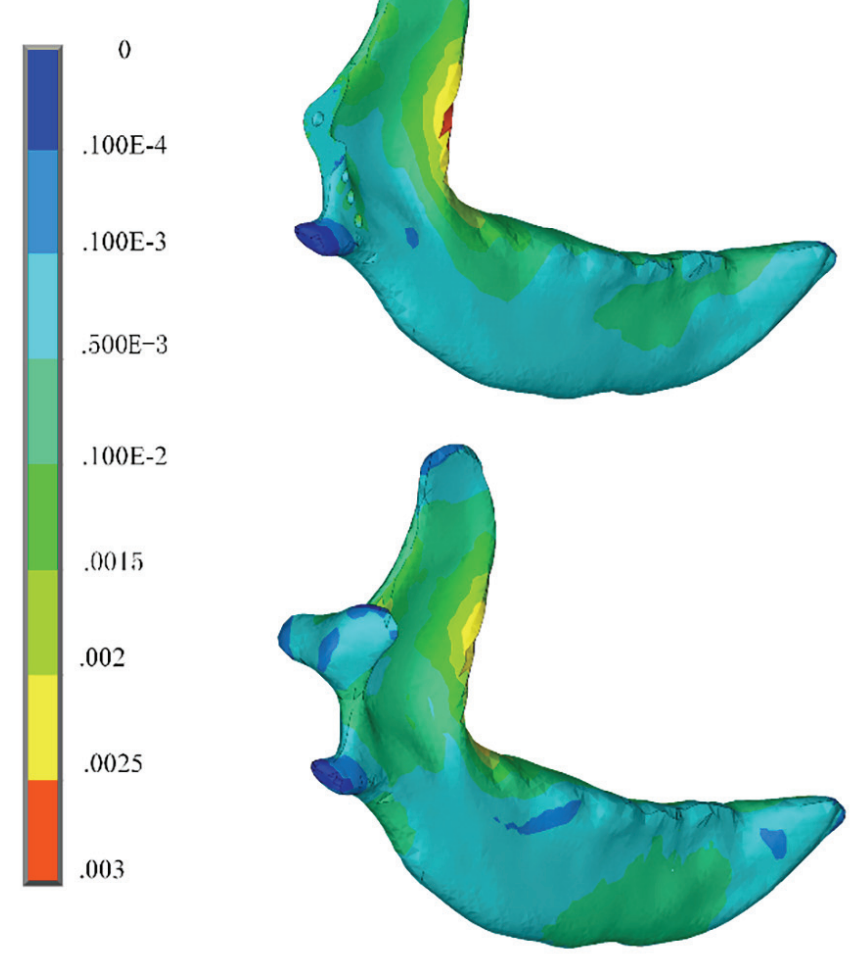

Right Mandible

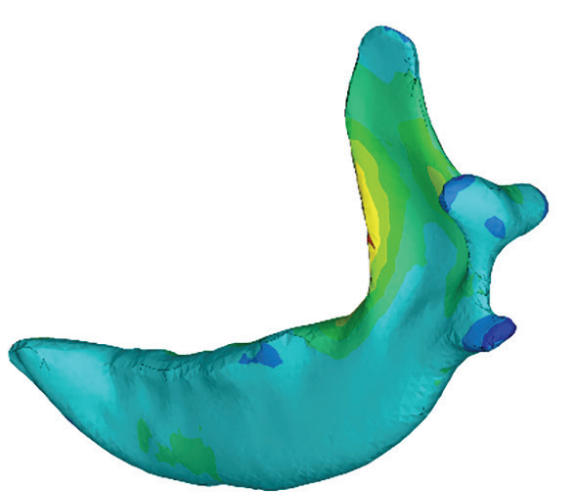

Model 1

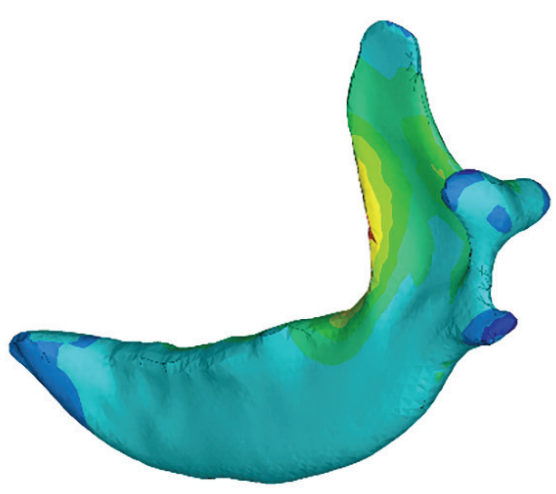

Model 2

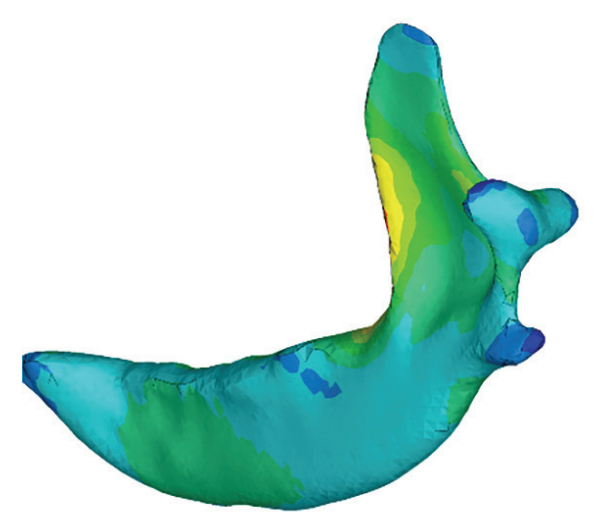

Left Mandible

Fig. 7. The maximum principal strain distributions of the mandibles in 3 models 

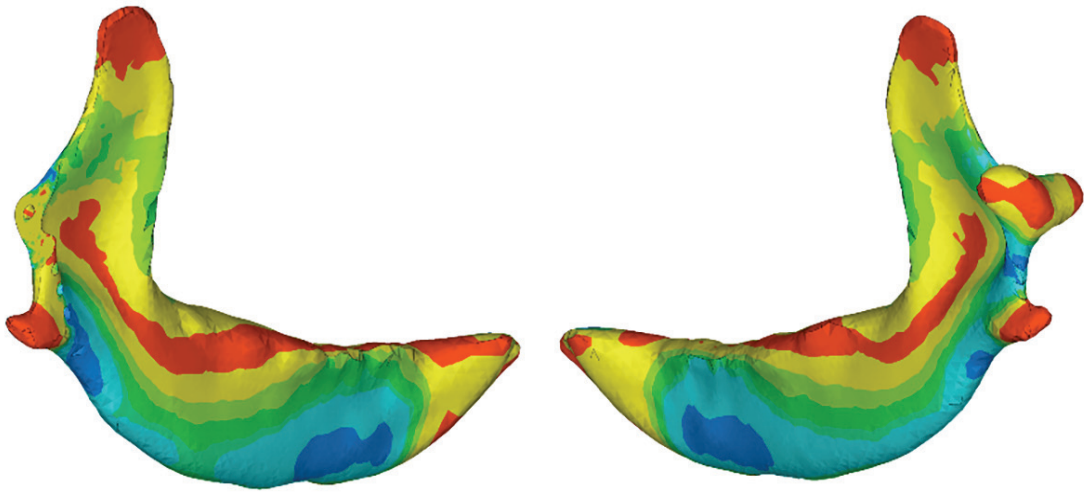

Model 1
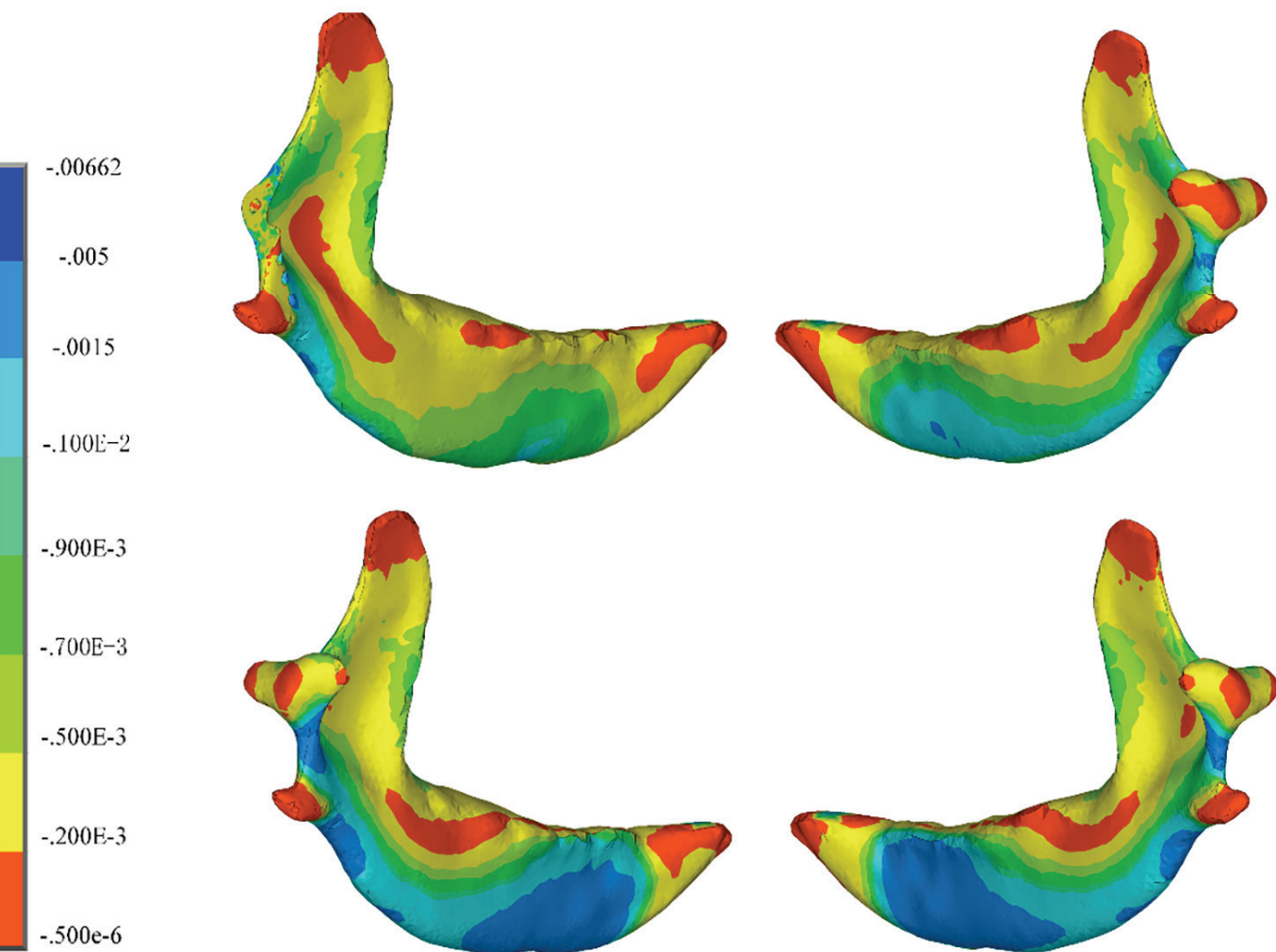

Model 2
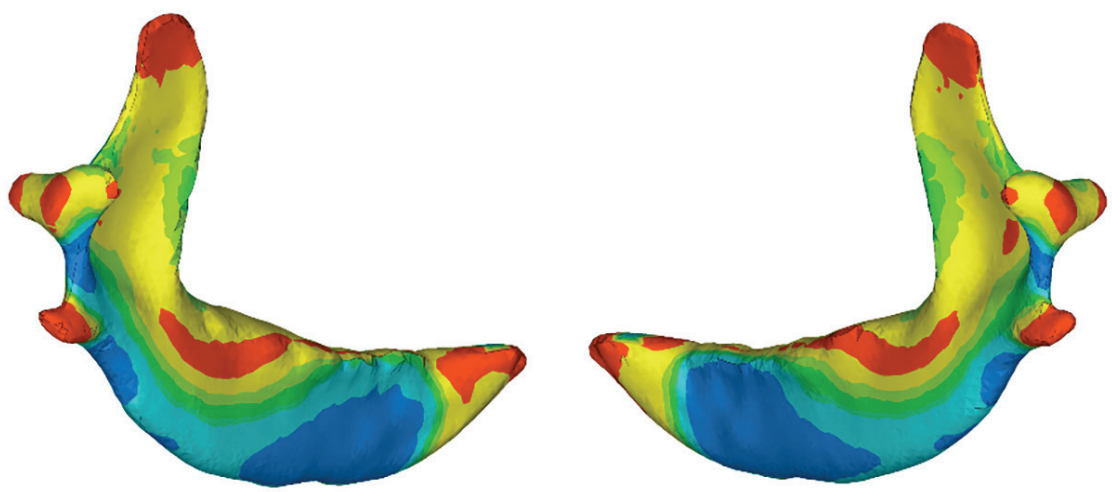

Model 3

Right Mandible

Left Mandible

Fig. 8. The minimum principal strain distributions of the mandibles in 3 models

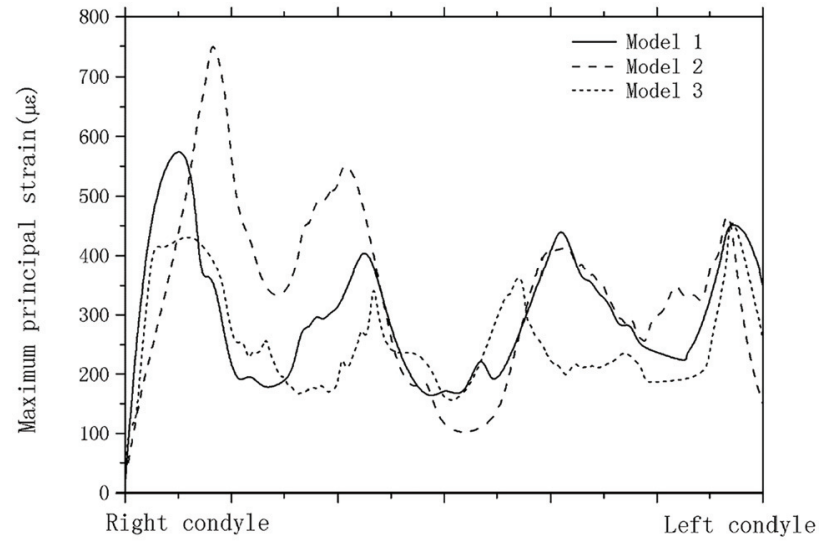

Fig. 9. The maximum principal strains on the external surface of the mandible along the line 1 in 3 models

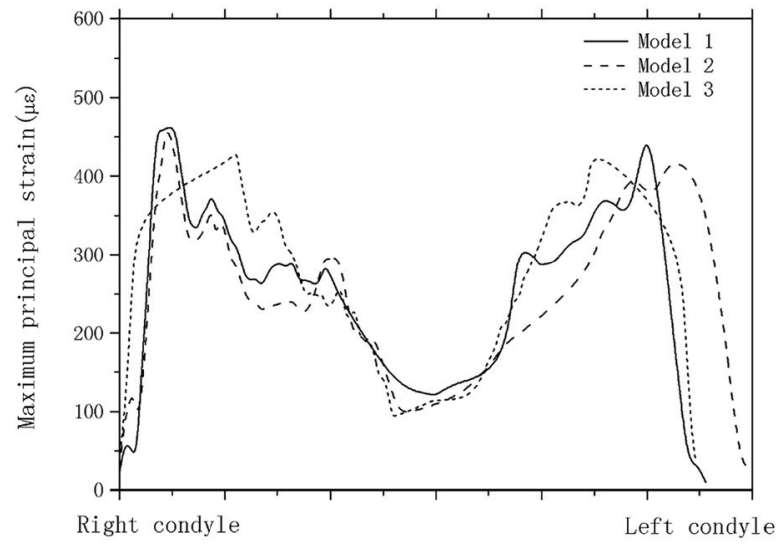

Fig. 10. The maximum principal strains on the external surface of the mandible along the line 2 in 3 models 


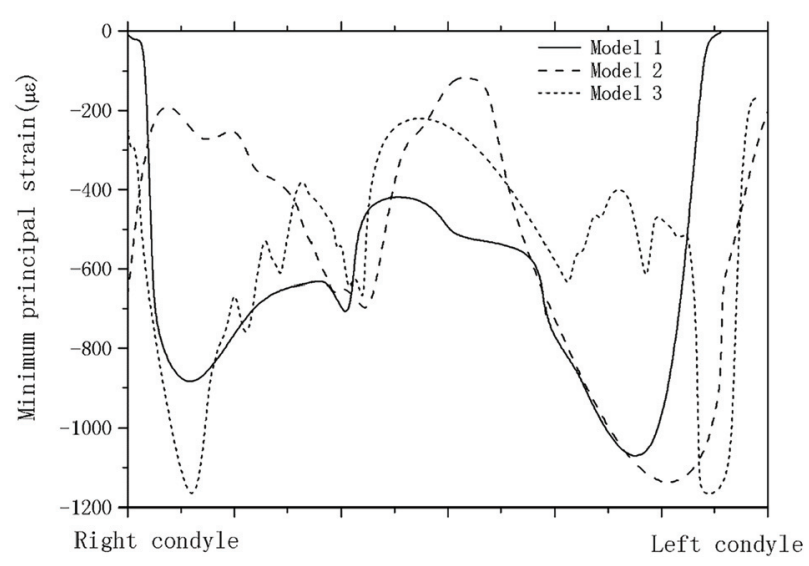

Fig. 11. The minimum principal strains on the external surface of the mandible along the line 1 in 3 models

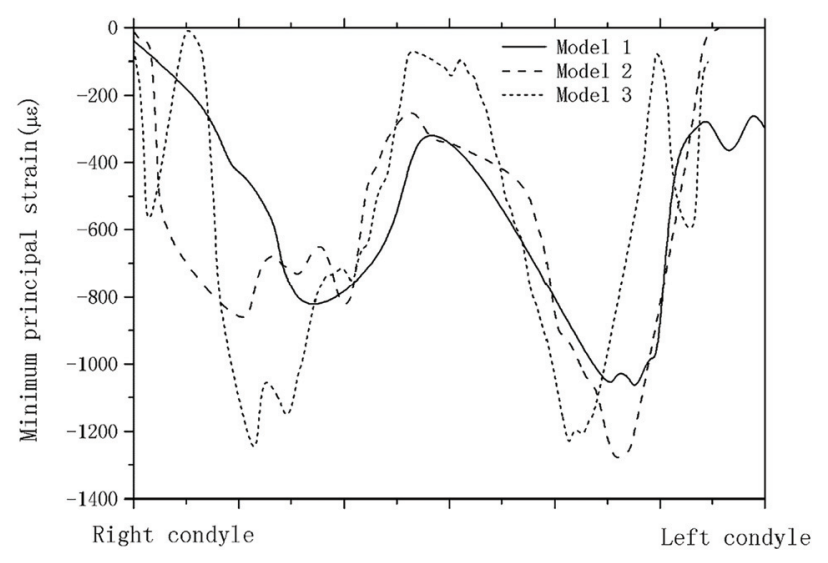

Fig. 12. The minimum principal strains on the external surface of the mandible along the line 2 in 3 models

\section{Discussion}

Biomechanical behaviors could affect the longterm results of TMJ prostheses. In this study, 3 models were established to compare the biomechanical response of the condyle prosthesis, the total TMJ prosthesis and the intact TMJ when muscle forces were applied. It could be inferred that different types of TMJ prostheses could cause different biomechanical effects on the bilateral TMJ regions and the prosthesis itself. The results revealed that the biomechanical behavior of the contralateral TMJ could be affected by the total TMJ prosthesis with customized morphology for the first time, which could be helpful for the TMJ prosthesis design and application.

The biomechanical behavior of 5 discs and 1 fossa component showed that Models 1 and 3 had the similar stress distribution on the same region of the right disc, which was close to the results of previous stud- ies [7], [23], [24]. This result could suggest that the customized condyle prosthesis in this study did not change the disc stress so much when the muscle forces were applied. It also could be inferred that the materials used in the condyle prosthesis to replace the original condyle did not lead to the increased stress of the disc and stress distribution change, even if a kind of metal was used. The morphology of the condyle prosthesis might be an influential factor for biomechanical responses of TMJs. Therefore, the customized profile would be recommended when the condyle prosthesis was applied.

The movements of TMJ were finished by bilateral TMJs at the same time. Changes in one side could definitely affect the other side under both physical and pathological status. Tanaka et al. reported that unilateral disc displacement could influence the stress in the contralateral joint using FEA [22]. Therefore, from the biomechanical view, the less the prosthesis affects the contralateral joint, the better. In this study, the condyle prosthesis in Model 1 had nearly kept the same stress of contralateral disc as the intact mandible in Model 3. This result showed that the condyle prosthesis in this study had good biomechanical behavior when muscle forces were applied at closed position.

In Model 3, the stress on the right disc was larger than the left disc. The reason for this might be the asymmetric profile between the two sides of the mandible. Some authors found that mandibular morphology was related to EMG activity and TMJ disc displacement [9], [15]. We applied the muscle forces of the same magnitude on bilateral mandible, which could cause some bias of the results. In this study, the main purpose was to compare the biomechanical effect of the same sides of the mandibular under the same mechanical environment. The stress distributions of discs in Models 1 and 3 were similar, for the left and the right side, respectively, which showed that the condyle prosthesis in this study restored the function of the defect of the right condyle.

The stress of the condyle prosthesis on Model 1 was less than the condyle component of the TMJ prosthesis on Model 2, which indicated that total TMJ prosthesis suffered larger stress than the condyle prosthesis, while the contralateral side of the total TMJ prosthesis had its stress changed and reduced by $12 \%$. It could be inferred that the total TMJ prosthesis changed the biomechanical behavior of the bilateral TMJs. Therefore, it should be considered when the total TMJ prosthesis was applied on the patient, especially for the contralateral TMJ. The change of the loads might cause pathological status 
[10]. We designed the condyle prosthesis and the total TMJ prosthesis according to the profile of the CT image of the dog, and applied the same muscle forces. The reason for the biomechanical changes could be inferred was that the materials of the prosthesis might lead to those results. UHMWPE has much larger Young's modulus than the disc. It is harder than biological disc to change its morphology when muscle forces were applied. Therefore, it changed the biomechanical effect of the contralateral TMJ when the prosthesis was applied.

According to the results, the anterior border of the condyle could be a stress concentration part, not only for the intact condyle, but also for the condyle prosthesis and the total TMJ prosthesis. This result is consistent with our previous study [28]. When the anterior part of mandible was loaded by the force, the anterior border and the lateral surface of the condyle was stressed more than other parts. This stress distribution style might be related to "L" shape of mandible. When the mandibular body was loaded, the anterior border of the condyle was more stressed than other parts, which should be considered when the prosthesis was applied for replacement of the condyle. The strain along line 2 among 3 models varied slightly, which suggested that the lower border of the mandible was affected little by the prosthesis.

FEA has been used for TMJ prosthesis evaluation in several studies, which supplied valuable information for the prosthesis improvement [9], [16], [20], [28], [29]. In this study, the stress distributions of the contralateral TMJs for unilateral TMJ replacement of customized condyle prosthesis and the total TMJ prosthesis were compared under the same biomechanical condition. In vivo experiments are to be performed to check the effectiveness of both prostheses and compare contralateral TMJ histological changes.

The main limitation of our research was the animal model, which was different from the human model. The aim of the study was to compare the biomechanical behavior between condyle prosthesis and total TMJ prosthesis. To keep the same biomechanical environment around the prosthesis was the essential factor for the comparison. Therefore, the animal models with the consistent biomechanical conditions could reveal the valuable comparison results. Another shortcoming was that this research belonged to theoretical study, which was different from experiments in reality. We plan to perform in vivo experiments to check the effectiveness and compare contralateral TMJ changes in future.

\section{Conclusions}

The comparison among the condyle prosthesis replacing the right condyle, the total TMJ prosthesis replacing the right TMJ and the intact TMJ showed that the condyle prosthesis with customized profile in this study had good biomechanical behavior and did not affect contralateral TMJ much. The materials with high Young's modulus in total TMJ prosthesis affected the biomechanical behaviors of bilateral TMJ, even with customized morphology. Therefore, elasticity of the TMJ prosthesis should also be considered in the future besides the customized-made morphology. The contralateral intact TMJ might be affected when the TMJ prosthesis was applied, which would bring the changes of the mandible biomechanical behavior.

\section{Acknowledgements}

This work was supported by the National Key R \& D Program of China (No. 2019YFB1706900), Peking University Clinical Scientist Program (No. BMU2019LCKXJ009), the Fundamental Research Funds for the Central Universities and Key Projects of International Scientific and Technological Innovation Cooperation among Governments under National Key R \& D Plan (No. 2017YFE0124500).

\section{Conflicts of Interest}

The authors confirm that there is no conflict of interest in relation to this work.

\section{Ethical approval}

The protocol of this study was approved by the Peking University Institutional Review Board (LA2014244). The guidelines for the care and use of laboratory animals of the Ministry of Science and Technology of the People's Republic of China were observed.

\section{References}

[1] Beek M., Aarnts M.P., Koolstra J.H., Feilzer A.J., van EIJDEN T.M., Dynamic properties of the human temporomandibular joint disc, J. Dent. Res., 2001, 80, 876-880.

[2] Conci R.A., Tomazi F.H., Noritomi P.Y., DA S.J., Fritscher G.G., Heitz C., Comparison of Neck Screw and Conventional Fixation Techniques in Mandibular Condyle Fractures Using 3-Dimensional Finite Element Analysis, J. Oral Maxillofac. Surg., 2015, 73, 1321-1327. 
[3] Del P.A., Doblare M., 3D finite element simulation of the opening movement of the mandible in healthy and pathologic situations, J. Biomech. Eng., 2006, 128, 242-249.

[4] Driemel O., Braun S., Muller-Richter U.D. et al., Historical development of alloplastic temporomandibular joint replacement after 1945 and state of the art, Int. J. Oral Maxillofac. Surg., 2009, 38, 909-920.

[5] Fricton J.R., LOOK J.O., SchiffMAn E., Swift J., Long-term study of temporomandibular joint surgery with alloplastic implants compared with nonimplant surgery and nonsurgical rehabilitation for painful temporomandibular joint disc displacement, J. Oral Maxillofac. Surg., 2002, 60, 1400-1412.

[6] Guarda-Nardini L., Manfredini D., Ferronato G., Temporomandibular joint total replacement prosthesis: current knowledge and considerations for the future, Int. J. Oral Maxillofac. Surg., 2008, 37, 103-110.

[7] Hattori-Hara E., Mitsui S.N., Mori H. et al., The influence of unilateral disc displacement on stress in the contralateral joint with a normally positioned disc in a human temporomandibular joint: an analytic approach using the finite element method, J. Craniomaxillofac. Surg., 2014, 42, 2018-2024.

[8] Hu K., Zhou J., Liu H., Hu M., Wang D., Rong Q., FAnG J., Development of the three-dimensional finite element model of adult dog temporomandibular joint in functional loading, Acad. J. PL A Postgrad. Med. Sch., 1997, 167-169.

[9] JEON D.M., Jung W.S., MAH S.J., KIM T.W., AhN S.J., The effects of TMJ symptoms on skeletal morphology in orthodontic patients with TMJ disc displacement, Acta Odontol. Scand., 2014, 72, 776-782.

[10] JibiKi M., Shimoda S., NaKagawa Y., KaWASAKi K., ASADA K., ISHIBASHI K., Calcifications of the disc of the temporomandibular joint, J. Oral Pathol. Med., 1999, 28, 413-419.

[11] Kawahara H., Kawahara D., Hayakawa M., Tamai Y., KuREMOto T., MATSUDA S., Osseointegration under immediate loading: biomechanical stress-strain and bone formation - resorption, Implant Dent., 2003, 12, 61-68.

[12] Koolstra J.H., van EiJden T.M., Consequences of viscoelastic behavior in the human temporomandibular joint disc, J. Dent. Res., 2007, 86, 1198-1202.

[13] Korioth T.W., Romilly D.P., Hannam A.G., Three-dimensional finite element stress analysis of the dentate human mandible, Am. J. Phys. Anthropol., 1992, 88, 69-96.

[14] Lin A.W., Vapniarsky N., Cissell D.D., Verstraete F.J.M., LiN C.H., HATCHER D.C., ARZI B.. The Temporomandibular Joint of the Domestic Dog (Canis lupus familiaris) in Health and Disease, J. Comp. Path, 2018, 161, 55-67.

[15] Matsumoto R., IoI H., Goto T.K. et al., Relationship between the unilateral TMJ osteoarthritis/osteoarthrosis, mandibular asymmetry and the EMG activity of the masticatory muscles: a retrospective study, J. Oral Rehabil., 2010, 37, 85-92.

[16] Ramos A., Mesnard M., Christensen vs. Biomet Microfixation alloplastic TMJ implant: Are there improvements?
A numerical study, J. Craniomaxillofac. Surg., 2015, 43, 1398-1403.

[17] Ramos A., Mesnard M., Comparison of load transfers in TMJ replacement using a standard and a custom-made temporal component, J. Craniomaxillofac. Surg., 2014, 42 (8), 1766-1772.

[18] Ramos A., Mesnard M., Relvas C., Completo A., Simoes J.A., Theoretical assessment of an intramedullary condylar component versus screw fixation for the condylar component of a hemiarthroplasty alloplastic TMJ replacement system, J. Craniomaxillofac. Surg., 2014, 42, 169-174.

[19] Sanovich R., Mehta U., Abramowicz S., Widmer C., Dolwick M.F., Total alloplastic temporomandibular joint reconstruction using Biomet stock prostheses: the University of Florida experience, Int. J. Oral Maxillofac. Surg., 2014, 43, 1091-1095.

[20] Shu J., Wang Q., Ma H., Teng H., Zheng T., Liu Z., Biomechanical study on the changes of stress in temporomandibular 2 joints after the orthognathic surgery in patients with mandibular 3 prognathism: a 3D finite element study, Acta Bioeng. Biomech., 2020, 22 (2), 155-163.

[21] Tanaka E., del Pozo R., Tanaka M., Tanne K., Threedimensional finite element analysis of human temporomandibular joint with and without disc displacement during jaw opening, Med. Eng. Phys., 2004, 26 (6), 503-511.

[22] TANAKA E., van EIJDEN T., Biomechanical behavior of the temporomandibular joint disc, Crit. Rev. Oral Biol. Med., 2003, 14, 138-150.

[23] TANne K., TANaka E., SAKuda M., The elastic modulus of the temporomandibular joint disc from adult dogs, J. Dent. Res., 1991, 70, 1545-1548.

[24] Teng S., Xu Y., Cheng M., Li Y., Biomechanical properties and collagen fiber orientation of temporomandibular joint discs in dogs: 2. Tensile mechanical properties of the discs, J. Craniomandib. Disord., 1991, 5, 107-114.

[25] Van LoON J.P., de BONT G.M., Boering G., Evaluation of temporomandibular joint prostheses: review of the literature from 1946 to 1994 and implications for future prosthesis designs, J. Oral Maxillofac. Surg., 1995, 53, 984-997.

[26] Vega L.G., Gonzalez-Garcia R., Louis P.J., Reconstruction of acquired temporomandibular joint defects, Oral Maxillofac. Surg. Clin. North Am., 2013, 25, 251-269.

[27] Westermark A., Heden P., Aagaard E., Cornelius C.P., The use of TMJ Concepts prostheses to reconstruct patients with major temporomandibular joint and mandibular defects, Int. J. Oral Maxillofac. Surg., 2011, 40, 487-496.

[28] Xu X., LuO D., Guo C., Rong Q., A custom-made temporomandibular joint prosthesis for fabrication by selective laser melting: Finite element analysis, Med. Eng. Phys., 2017, 46, 1-11.

[29] Xu X., Rong Q., Guo C., Study of protection factors of skull base with loaded mandible, China J. Oral Maxillofac. Surg., 2011, 1, 1-7. 